\title{
Water Flow Based Vessel Detection in Retinal Images
}

\author{
Xin U Liu, Mark S Nixon \\ ISIS group, School of ECS, University of Southampton, Southampton, U.K. \\ \{Xl104, M.S.Nixon\} decs.soton.ac.uk
}

Keywords: feature extraction, water flow, snake, vessel

\begin{abstract}
Previous vessel segmentation methods mainly concentrate on the general structure, and often ignore the accuracy, smoothness and continuity of vessel boundaries. A water flow based method is proposed to solve the problem. It embodies the fluidity of water and hence can handle the complex topological changes of vessels. A snake-like force functional combining edge-based and region-based forces produces capability for both accuracy and range. Properties analogous to surface tension and adhesion are also applied so that the smoothness of the evolving contour and the ability to flow into narrow branches can be controlled. The technique has been assessed on synthetic and real images, and shows excellent detection performance and ability to handle noise.
\end{abstract}

\section{Introduction}

Accurate vessel structure analysis plays an important role in medical imaging since it is needed in many diagnoses like diabetic retinopathy and hypertension, where information such as vessel width, tortuosity and abnormal branching is required. Manual delineation of the vessels is ineffective especially when the number of images is large. Therefore, automatic analysis is needed.

Previous methods can be classified into two groups: modelbased techniques and supervised ones. The former methods first set up certain criteria of vessels and then find the pixel response to these standards. Morphological properties such as width, linearity, connectivity, and Gaussian-like profile, together with the pixel attributes like intensity and gradient, are often used as the criteria. Finally, some identification processes are applied. The techniques include vessel tracking [10], morphological filtering [12], and locally adaptive thresholding [5,6]. Alternatively, supervised methods compute a feature vector for each pixel: Staal et al [11] compute it using the intrinsic property that vessels are elongated structures; Leandro et al [8] construct the vector by colour information and the wavelet transform response. The feature vectors are then classified by supervised classifiers which require manually labelled images for training.

Those methods primarily concentrate on the general vessel structure and ignore the accuracy, smoothness and especially the continuity of the boundaries. Active contours (snakes) can tackle these problems by considering an object boundary as a single, connected structure, and applying certain smooth-ness constraints. In vessel extraction, however, the classical snake is of limited use as it needs good initialization near the boundary and cannot handle topological changes. Snake energy functionals using region statistics or likelihood information [3, 4] have been proposed to overcome the initialization restriction. A common premise is to increase the capture range of the external forces to guide the curve towards the boundaries. For complex topology detection, several authors have proposed adaptive methods like the Tsnake [9] based on repeated sampling of the evolving contour on an affine grid, and geometric active contours [2] where the planar curve is represented as a level set of an appropriate 2D surface. Both methods detect complex shapes well, at the cost of significantly increased complexity.

This paper proposes a new feature extraction method based on water flow. Unlike the famous watershed method, which is based on mathematical morphology and is often combined with snakes [7] and region growing [1], the focus is now on the "water" itself rather than the "landscape" of images as the properties of water, like fluidity and surface tension, are suited to complex shape detection and hence vessel extraction. Combined by local region processing with binary decision processes, the technique has a low computation requirement. We first introduce the related physical principles and the framework of the method, and then define all the analogical factors. Finally, results both for synthetic and for real iris images are presented, which show the resolution of problems like topological changes, and good noise immunity.

\section{Methodology}

Water flow is a compromise between several factors: the position of the leading front of a water flow depends on pressure, surface tension, adhesion/capillarity. There are some other natural properties like turbulence and viscosity, which are ignored here. Image edges and some other characteristics that can be used to distinguish objects are treated as the "walls" terminating the flow. The final static shape of the water will describe the related object's contour.

The flow is determined by pressure and the resistance. The relationship between the flow rate $f_{r}$, the flow resistance $\mathcal{R}$ and the pressure difference, is given by:

$$
f_{r}=\left(P_{i}-P_{o}\right) / \mathcal{R}
$$

where $P_{\mathrm{i}}$ and $P_{\mathrm{o}}$ are pressure of the inflow and outflow, respectively. The pressure difference drives the flow and

$$
f_{r}=A V_{\text {effective }}
$$


where $A$ is the cross-sectional area and $\mathrm{V}_{\text {effective }}$ is the effective flow velocity. Hence the velocity can be related to force and resistance through equations (1) and (2).

There are small discontinuities or weak regions existent on the contours which may lead to "leakage" of water. Surface tension, which can form a water "film" to bridge gaps, is then applied to overcome the problem. An attractive force existing between water and walls, named adhesion is defined as the attractive force generated by image edges. It is adopted in the new technique to assist surface tension to bridge edge gaps and allow flow into narrow branches.

\subsection{Framework of the operator}

The new method has little dependence on the starting contour shape. The only limitation on initialization is that it cannot cross the target object's boundaries. One pixel in the image is considered to be one basic unit of the water, and the pressure between an element and each of its neighbors is assumed to be the same. An adaptive source is assumed so that the water can keep flowing until stasis, where flow ceases. An inner element with symmetrical distribution of neighbors hence suffers zero resultant pressure. A water contour element, however, has asymmetrically distributed neighbors (and possibly an additional adhesive force), thus has non-zero pressure difference which leads to a non-zero velocity by equations (1) and (2), and can move outwards. Hence only boundary elements are of interest.

The flow process is assumed to be made up of two separable steps. The first stage is acceleration: the contour element achieves a velocity due to the presence of the pressure difference (and any adhesive force). The next step is external movement where the moving element is now free from the influence from other water elements and suffers only external image forces. This is not consistent with the real flow process but is sufficient for the digital image analogy and greatly simplifies the algorithm.

The water element can move outwards in any direction for which the component of velocity is positive. However, only if the velocity in the direction is sufficiently large, can the element break through the image resistant forces and reach the new position. To reconcile the flow velocity with forces, dynamical formulae are used. We may compute the displacement of a contour element on each possible direction within a fixed time interval, which is similar to snake techniques. However, for simplicity and avoiding the interpolation problem, a framework like region growing and the greedy snake is used: the element will flow to some positions if certain conditions or formulae are satisfied. Here, an equation describing the conservation of energy is employed. If assuming that an element, which has a positive velocity $v$ on a particular direction and is acted by the force $F$ during the process, can arrive at the direction-related position ultimately, then this equality must be fulfilled:

$$
m v_{F}^{2} / 2=F S+m v^{2} / 2
$$

where $v_{F}$ is the final scalar velocity after fixed displacement $S$ and $m$ is the assumed mass, respectively. Here force $F$ is a scalar which is positive when the force is consistent with velocity $v$, and negative otherwise. The summation on the right hand side is just the movement decision operator: only if $F$ is negative, can the summation be negative and thus the equality in equation (3) cannot be satisfied.

\subsection{Flow driving force with surface tension}

The flow driving force results from the pressure difference between water elements. Using a matrix $\mathbf{W}$ to save the water information where a water element has a value one and others are all zeros, then the gradient of $\mathbf{W}$ will give the information of pressure difference. As the potential flow in each direction will be examined separately, the component of each force is of interest. We have, for a particular direction $i$ :

$$
\mathbf{F}_{D, i}=-\nabla_{i} \mathbf{W}
$$

where $\nabla_{i}$ is the component gradient in direction $i$ and $\mathbf{F}_{D, i}$ is the corresponding matrix saving the forces, respectively. We use a negative gradient when the gradient points from lower intensity (non-water) to higher one (water), and the driving force acts oppositely. Therefore, the driving force strength on direction $i$ at point $(x, y)$ is just the $(x, y)$ th entry of $\mathbf{F}_{D, i}$.

From physics, the surface tension is decided by the temperature and the water itself. In this image analogy, it is defined as a constant attractive force between the contour elements. So in the previous convolution, we can just modify the water matrix with the contour position information so that the point will exhibit attractive forces.

This is done by setting contour elements entries in $\mathbf{W}$ as fixed negative values, like $-t$. Then, replacing $\mathbf{W}$ in equation (5) with the new matrix $\mathbf{W}^{\prime}$ ' (note that the force should now be normalized by the possible maximum of $3+2 t$ ), will give the driving force combined with surface tension. Here, we set $t=1$.

\subsection{Resistance to flow and the velocity}

From equation (1) and (2), the flow velocity is inversely proportional to the resistance of water. In a physical model, the flow resistance is decided by the water, the flow channel and temperature etc. Since this is a physical analogy which offers great freedom in selection of parameter definitions, we can assign high resistance values for unwanted image attributes and low values to preferred ones. For instance, in iris vessel detection, if the vessels have relatively low intensity, we can define the resistance to be proportional to the intensity of the pixel. If we couple the resistance with the edge information, the process will become adaptive. That is, when the edge response is strong, resistance would be large and so the flow velocity would be weakened. According to equation (3), the movement decision will now be dominated by the force acting during the exterior movement. Thereby, even if the driving force set by users is too "strong", the resistance would lower its influence at edge positions and the problem in balloon models [1], where strong driving forces may overwhelm "weak" edges, can be suppressed. We first write an equation of velocity by (1) and (2):

$$
\mathbf{V}_{i}=\mathbf{F}_{i} / A \cdot \mathcal{R}
$$


where $\mathbf{V}_{i}$ is the resulting flow velocity. The direction of $\mathbf{V}$ is the same as the force $\mathbf{F}_{i}$. In this paper, $A$ is set as a constant, and $R$ at position $(u, v)$ is determined by

$$
\mathcal{R}(u, v)=\exp \{-k \cdot \mathbf{E}(u, v)\}
$$

where $\mathbf{E}$ is the edge response matrix and $k$ controls the fall of the exponential curve.

\subsection{Image forces}

The gradient of an edge response map is often defined as the potential force in active contour methods since it gives rise to vectors pointing to the edge lines [3]. This is also used here. The force is large only in the immediate vicinity of edges and always pointing towards them. The second property means that the forces at two sides of an edge have opposite directions. Thus it will attract water elements onto edges and prevent overflow. The potential force on a contour element $\left(x_{c}, y_{c}\right)$ is given by:

$$
\mathbf{F}_{P}=\nabla \mathbf{E}\left(x_{t}, y_{t}\right)
$$

where $\nabla \mathbf{E}$ is the gradient of the edge map, and $\left(x_{t}, y_{t}\right)$ are the coordinates of the flow target because the potential force is presumed to act during the second stage of flow where the element has left the contour and is moving to the target.

Adhesion is defined as the attraction between water and adjacent vessel walls in physics. In the image analogy, it is determined by potential force based on an edge map with "flooded" positions set to zero. In this map, the edges that have been occupied by the water are ignored so that the edges are clipped. As water flows, vectors (forces) pointing from the flooded edges to the existent ones are generated iteratively and thus assist in flow to the reserved edge lines. The adhesion at contour point $\left(x_{c}, y_{c}\right)$ is defined as

$$
\mathbf{F}_{A}=\nabla \mathbf{D}\left(x_{c}, y_{c}\right)
$$

where $\mathbf{D}$ is the edge map eroded by the flowing water. This equation effectively defines the attractive force from edges to the water. Therefore, even if the water has flowed onto an edge point, it can still move to the adjacent edges. This will thus help water flow into narrow branches, and "flood" small noise pixel clusters to give noise robustness.

The forces defined above work well as long as the gradient of edges pointing to the boundary is correct and meaningful. However, as with corners, the gradient can sometimes provide useless or even incorrect information. Unlike the method used in the inflation force [1] and T-snake [6], where the evolution is turned off when the intensity is bigger than some threshold, we propose a pixel-wise regional statistics based image force. The statistics of the region inside and outside the contour are considered respectively and thus yield a new image force:

$$
F_{S}=-\frac{n_{\text {int }}}{n_{\text {int }}+1}\left(\mathbf{I}\left(x_{t}, y_{t}\right)-\mu_{\text {int }}\right)^{2}+\frac{n_{\text {ext }}}{n_{\text {ext }}-1}\left(\mathbf{I}\left(x_{t}, y_{t}\right)-\mu_{e x t}\right)^{2}
$$

where subscripts "int" and "ext" denote inner and outer parts of the water, respectively; $\mu$ and $n$ are the mean intensity and number of pixels of each area, separately; $\mathbf{I}$ is the original image. The equation is deduced from the Mumford-Shah functional used in [5]:

$$
\begin{aligned}
F_{1}(C)+F_{2}(C)=\int_{\text {inside }(C)}\left|I(x, y)-\mu_{\text {int }}\right|^{2} & \\
& +\int_{\text {outside }(C)}\left|I(x, y)-\mu_{\text {ext }}\right|^{2}
\end{aligned}
$$

where $C$ is the closed evolving curve. If we assume $C_{0}$ is the real boundary of the object in the image, then when $C$ fits $C_{0}$, the term will achieve the minimum. Instead of globally minimizing the term as in [5], we obtain equation (9) by looking at the change of the total sum given by single movement of the water element. If an image pixel is flooded by water, the statistics of the two areas (water and non-water) will change. Both changes are derived and thus yield equation (9). The derivation is shown in the Appendix.

The edge-based potential forces can provide a good localization of the contour near the real boundaries (i.e., accuracy) but have very limited capture range and are not suitable for edge corners, whilst the region-based forces have a large basin of attraction but cannot provide good detection accuracy. The complementary properties motivate a unification of the two forces. A convex combination method is hence chosen and the combined force is given by:

$$
F=\alpha F_{P}+(1-\alpha) F_{S}
$$

where all terms are scalar quantities, and $\alpha(0 \leq \alpha \leq 1)$ is determined by the user to control the balance between them.

\subsection{Movement decision process}

Equation (3) has provided the inequality to determine the feasibility of flow. For each contour element, we have presented equations computing the driving force $\mathbf{F}_{\mathrm{D}}$ modified by surface tension, adhesion $\mathbf{F}_{\mathrm{A}}$ and resistance $\mathcal{R}$. Flow velocity $\mathbf{V}$ can then be obtained through equation (5). If the velocity points towards the exterior of the water, the element is assumed to leave the original position. A unified image force $\mathbf{F}$ provided by equations (7) and (11) is then turned on. The summation in equation (3) can be computed and the sign determines the result of the movement.

Defining $m$ and $S$ in equation (3) as constants, we can then present the new and detailed expression with parameters defined before:

$$
J=\lambda\left(\left(F_{D}+F_{A}\right) / \mathcal{R}(x, y)\right)^{2}+F
$$

where $\lambda$ is a regularization parameter set by users which controls the tradeoff between the two energy terms. It can be considered to be determined by the combination of mass $m$, displacement $S$ and area $A$. Its value reflects smoothing of image noise. For example, more noise requires larger $\lambda . F_{A}$ and $F_{D}$ are the scalar components on the movement direction of $\mathbf{F}_{\mathrm{A}}$ and $\mathbf{F}_{\mathrm{D}}$, respectively. A positive direction is defined from the origin to the target. The movement decision can be completely made by this operator since the term on the right hand side inside the brackets gives the velocity information and $J$ corresponds to the ultimate kinetic energy. If the velocity component is greater than zero and if $J$ is positive, the movement is said to be feasible and the target point will be flooded by water. 


\section{Experimental results}

The new technique is applied to both synthetic and natural iris images, and noise analysis is also performed.

\subsection{Synthetic images}

A $512 \times 512$ test image was generated for performance evaluation according to chosen criteria: a) horizontal, vertical and diagonal branches are included; b) narrow and wide branches are presented, respectively; c) there is a circular pipe so that we get a curve with smoothly changing curvature; d) each half of the object has a different intensity so that weak edges exist between them. The image is suited to assess the operator's ability in complex feature extraction. Noise immunity is tested by adding Gaussian and impulsive noise to the image. Figure 1 shows the evolution and the result.

The detection is successful in total, and the immunity to impulsive noise should be emphasized. It is very difficult for snakes and region growing approaches to deal with impulsive noise as the edge response is very strong. In figure 1(c) and (d), almost all the noise points inside the object are flooded, and the detected contour is reasonably accurate. The robustness to impulsive noise arises from the fluidity and the adhesion: water surrounds the small clusters of impulsive noise pixels, and the adhesive force given by the noise pixels attracts the water to flow inside the noise area. Thereby, unless the noise clusters are too large, the noise pixels will be flooded. The most significant failure shown by figure 1d) is the incomplete detection of the thinnest diagonal branch in the top right corner. This is because the branch is too narrow and a noise cluster "blocks" the pipe. In practical applications, this kind of gap will exist and makes the contour extraction to

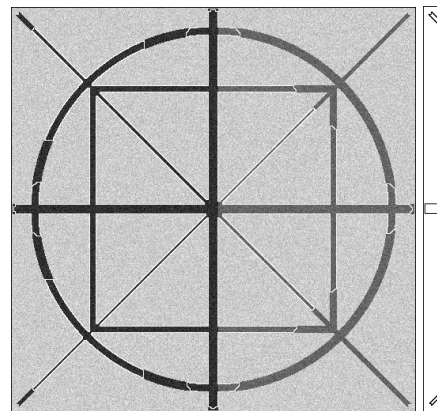

(a) evolving contour

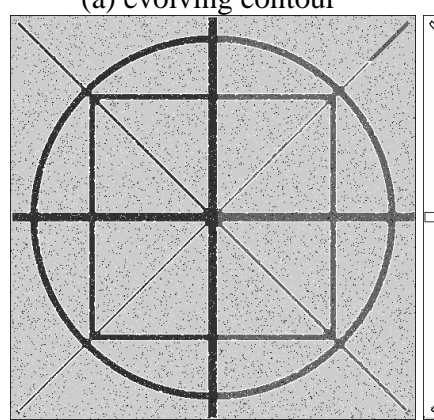

(c) evolving contour

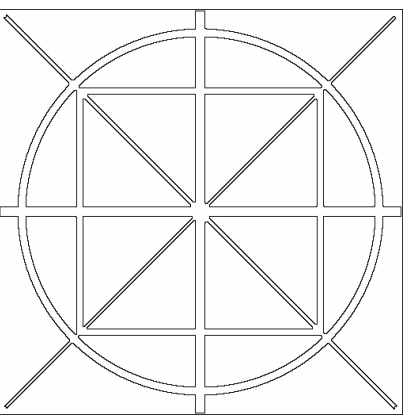

(b) final result

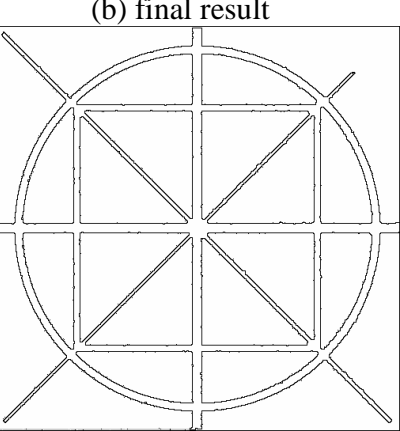

(d) final result
Figure 1: Flow progress for test image contaminated by $10 \%$ Gaussian noise ( $\mathrm{a}$ and $\mathrm{b}$ ) and $5 \%$ impulsive noise (c and d).

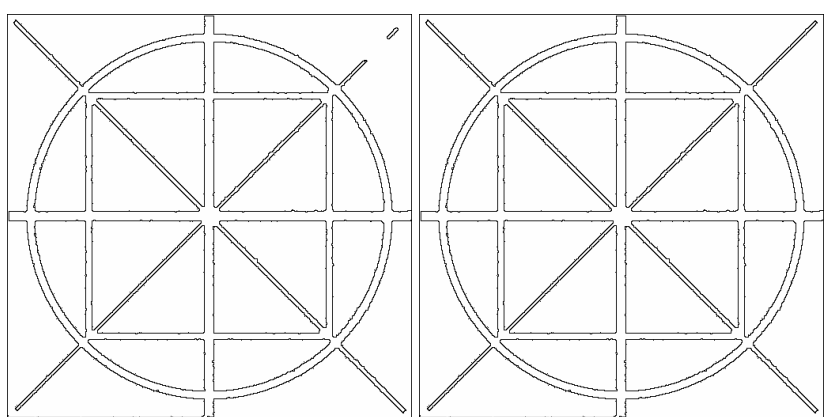

Figure 2: A better result for impulsive noise case achieved by adding one further water source.

terminate early. Flow from multiple sources can be considered, to overcome the problem. In this simple case, as shown in figure 2 , a new source is initialized inside the undetected area. It then fills the region and merges with the "main" part. Therefore a complete detection can be achieved.

\subsection{Vessel detection in retinal images}

Based on the eye fundus photos which are acquired by digital camera and are stored as true colour images, the performance of the operator in vessel detection is studied. The green channel, which is generally accepted as the one providing the greatest boundary information of vessels $[1,2,3]$, is used here. Except for the difficulties brought by the irregular shapes of vessels, the images themselves have some problems as well, e.g., noise, uneven brightness and contrast (the closer to the centre, the better the quality), and some vessel features have very low contrast or inhomogeneous intensity. Therefore, image enhancement techniques are often used to improve the image quality before detection, but the process also increases the noise level. The water-flow method, however, is relatively immune to noise and hence can incorporate with image enhancement for pre-processing.

The flow is initialized at a point inside a vessel branch and ended at stasis. Figure 3 shows the progress. We can see that the new technique can handle problems like complex topological changes and noise. Moreover, the detected vessel is a single, connected structure which is superior to the previous algorithms.

From figure 3(d), we can see that the detection is not yet complete. Some of the vessels are not detected. The failure is due to the initialization restriction (vessels without existing connection to the detected "vessel system" will certainly be missed), or to the gaps in the branches. Some of the gaps are formed naturally and are meaningful as the ones formed by intersection of two vessels. The other gaps result from imaging factors such as the camera, lighting, and noise etc. To handle these problems, multi-source initialization, as in section 3.1, will be helpful. To show this, two new water "sources" have been added as indicated by figure 3(e), and the final result is shown in figure 3(f). The water from the upper source merged with the previously detected vessel structure which gives a more complete result. However, the lower one still stops at gaps. In that case, some gap-linking techniques could be applied to obtain continuous contours but 


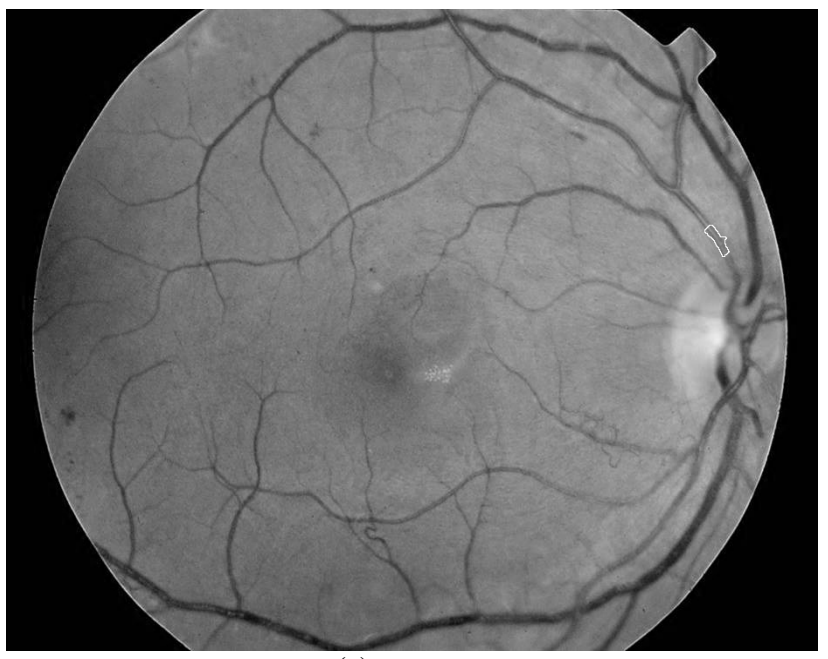

(a)

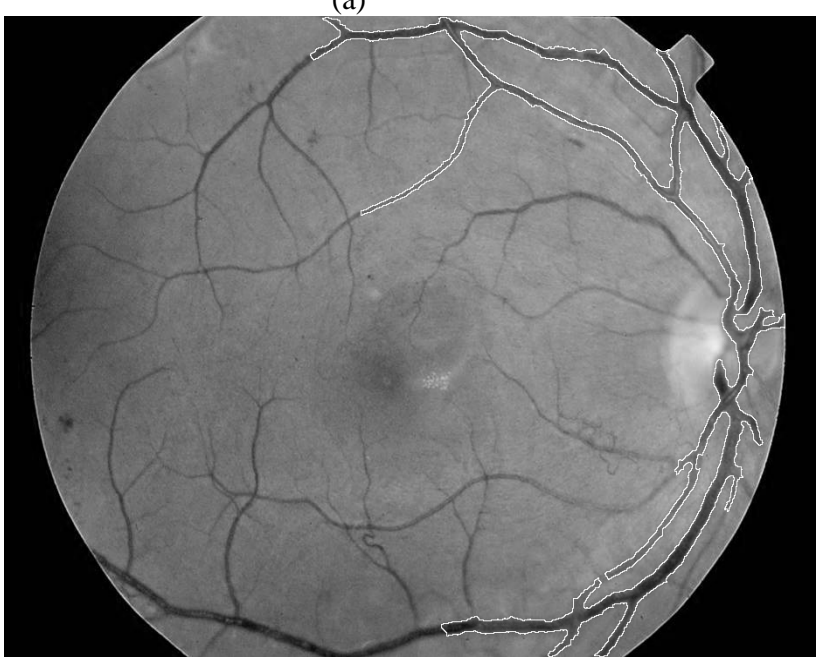

(c)

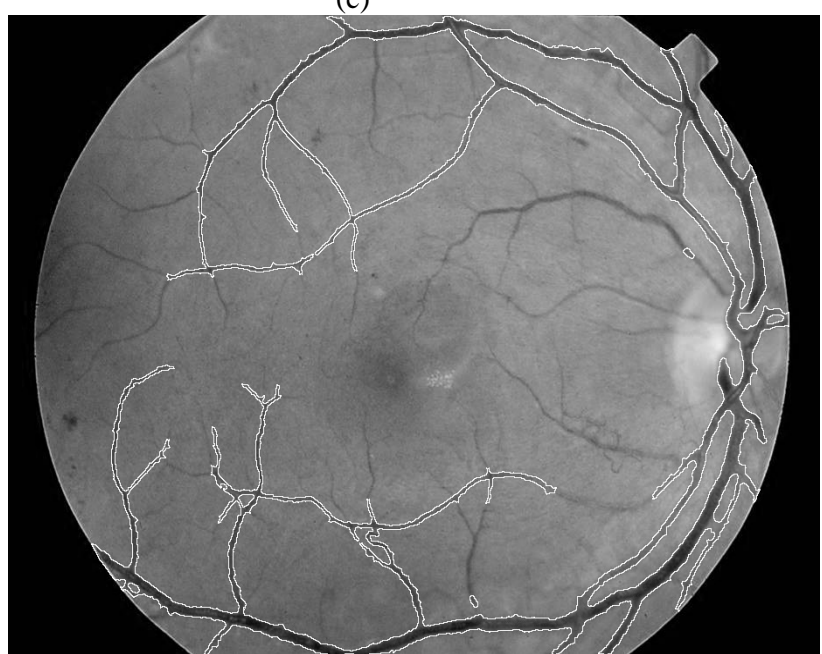

(e)

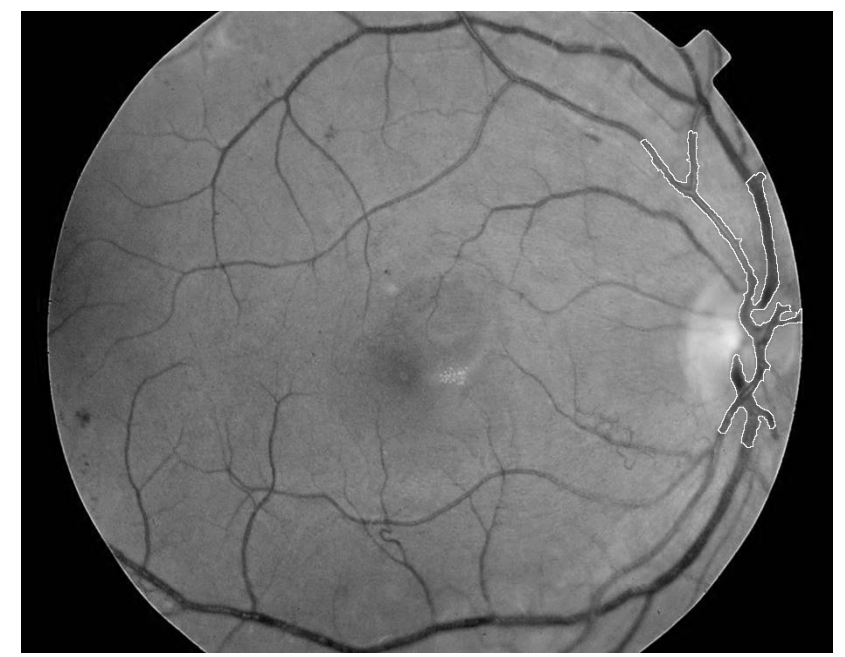

(b)

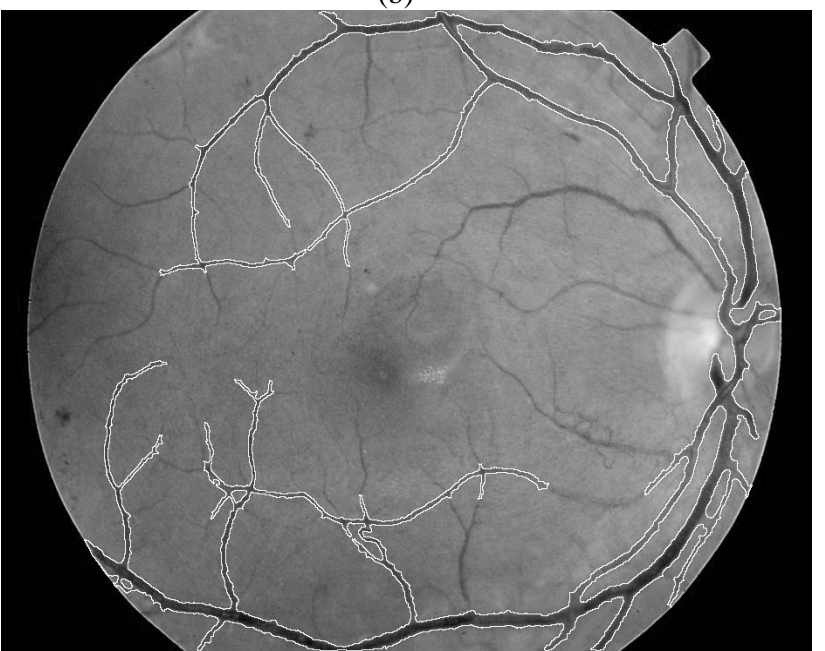

(d)

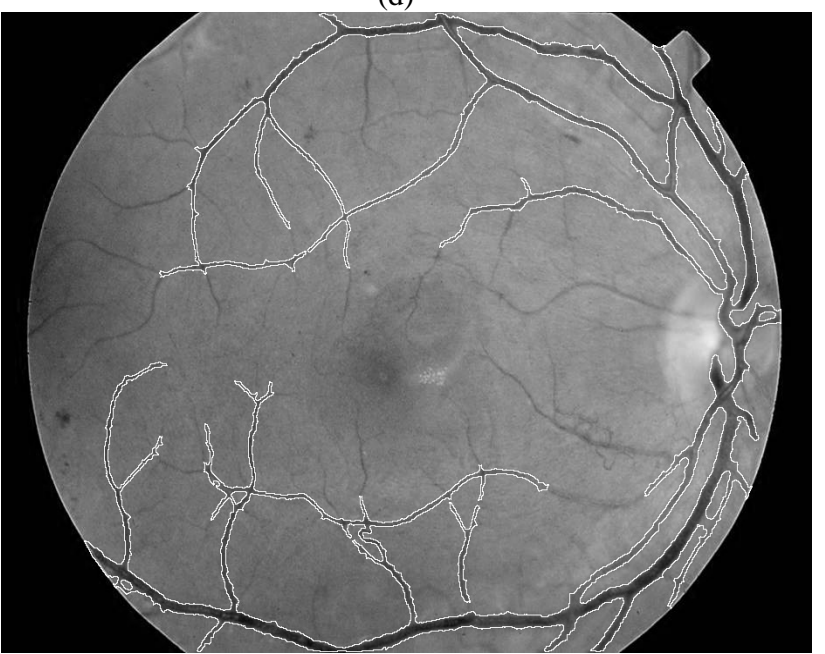

(f)

Figure 3: Vessel detection results where $\alpha=0.5, \lambda=1, k=50$. : (a) - (d) indicates the flow from one branch to all the other connected vessels; two new water sources are added in (e) and (f) gives the final result with the three source

this will be studied in further applications. Automated multisource initialization methods are often incorporated with techniques like region growing and are not invoked here.

\section{Conclusions and Future work}

This paper introduces a new water flow based feature extraction method, which successfully realizes the significant attributes of flow process: the fluidity, the surface tension and 
the adhesion. The resistant force given by images is defined by a combination of object boundary and regional information. The problems of topological changes are settled whilst the attractive features of snakes like the smooth and continuous contour is retained. Noise immunity is also tested and the results are encouraging. Besides, the complexity is relatively low and hence is expected to be of potential use in practical applications. Here the application to vessel detection, where the previous algorithms often generate discontinuous and irregular contours, is studied and the detection is successful. A more thorough performance evaluation based on large datasets with possible extensions such as multi-seeded flow and gap-linking will be studied in future work.

\section{References}

[1] A. Bleau and L. J. Leon, "Watershed-base segmentation and region merging," CVIU, 77: 317-370, 2000.

[2] V. Casselles, R. Kimmel, and G. Spiro, "Geodesic active contours," Int'l Journal of Computer Vision, 22(1):61-79.

[3] T. F. Chan and L. A. Vese, "Active contours without edges," IEEE Trans. Img. Proc., 10: 266-276, 2001.

[4] M. Figueiredo and J. Leitao, "Bayesian estimation of ventricular contours in angiographic images," IEEE Trans. Medical Imaging, 11: 416-429, 1992.

[5] A. Hoover, V. Kouznetsova, and M. Goldbaum, "Locating blood vessels in retinal images by piecewise threshold probing of matched filter response,"IEEE trans. Medical Imaging., 19(3): 203-210, 2000.

[6] X. Jiang and D Mojon, "Adaptive local thresholding by verification-based multithreshold probing with application to vessel detection in retinal images," IEEE trans. PAMI, 25(1): 131-137, 2003.

[7] V. Kiran, P. K. Bora, "Watersnake: integrating the watershed and the active contour algorithms," Proc. of TENCON`03, 2: 868-871, 2003.

[8] J. Leandro, J. Soares, R. Cesar, and H. Jelinek, "Blood vessels segmentation in non-mydriatic images using wavelets and statistical classifiers," Proceedings of SIBGRAPI'03, pp. 262-269, 2003.

[9] T. McInerney and D. Terzopoulos, "Topologically adaptive snakes," ICCV95, pp. 840-845, 1995.

[10]A. Pinz, S. Bernogger, P. Datlinger, and A. Kruger, "Mapping the human retina," IEEE trans. Med. Img., 17(4): 606-619, 1998.

[11] J. Staal, M. D. Abramoff, M. Niemeijer, M. A. Viergever, and B. Ginneken, "Ridge-based vessel segmentation in colour images of retina," IEEE trans. Med. Img., 23(4): 501-509, 2004.

[12]F. Zana and J. Klein, "Segmentation of vessel-like patterns using mathematical morphology and curvature evaluation," 10(7): 1010-1019, 2001

\section{Appendix}

The sums of integrations in equation (10) have to be modified due to the discrete nature of image pixels:

$$
F_{1}(C)+F_{2}(C)=\sum_{j=1}^{n_{\text {ext }}-1}\left(u_{j}-\mu_{\text {int } 0}\right)^{2}+\sum_{j=1}^{n_{\text {ext }}-1}\left(u_{j}-\mu_{\text {ext } 0}\right)^{2}
$$

where subscript " 0 " means the state before movement, and $u_{i}$ and $u_{j}$ represent pixels inside and outside the water region respectively. After a single pixel flow, the numbers become $n_{\text {int }}+1$ and $n_{\text {ext }}-1$, respectively with corresponding changes in the statistics. By denoting the flooded pixel as $u_{n}$, we can deduce the changes. For the external term, the new term is

$$
\begin{aligned}
F_{2}^{\prime}(C) & =\sum_{j=1}^{n_{e x t}-1}\left(u_{j}-\mu_{e x t 1}\right)^{2} \\
& =\sum_{j=1}^{n_{e x t}-1}\left(u_{j}-\frac{\mu_{e x t 0} \times n_{e x t}-u_{n}}{n_{e x t}-1}\right)^{2} \\
& =\sum_{j=1}^{n_{e x t}-1}\left(u_{j}-\frac{\mu_{e x t 0} \times\left(n_{e x t}-1\right)+\mu_{e x t 0}-u_{n}}{n_{e x t}-1}\right)^{2} \\
& =\sum_{j=1}^{n_{e x t}-1}\left(u_{j}-\mu_{e x t 0}-\frac{\mu_{e x t 0}-u_{n}}{n_{e x t}-1}\right)^{2} \\
& =\sum_{j=1}^{n_{e x t}-1}\left(u_{j}-\mu_{e x t 0}\right)^{2}+\left(n_{e x t}-1\right)\left(\frac{\mu_{e x t 0}-u_{n}}{n_{e x t}-1}\right)^{2} \\
& +2\left(\frac{u_{n}-\mu_{e x t 0}}{n_{e x t}-1}\right)_{j=1}^{n_{e x t}-1}\left(u_{j}-\mu_{e x t 0}\right)
\end{aligned}
$$

Denote $\left(u_{n}-\mu_{\text {ext } 0}\right)$ as $\Delta$, then $\sum_{j=1}^{n_{\text {exx }}-1}\left(u_{j}-\mu_{\text {ext } 0}\right)=-\Delta$ (since $\left.\sum_{j=1}^{n_{\text {ext }}}\left(u_{j}-\mu_{\text {ext } 0}\right)=0\right)$, and then we have:

$$
\begin{aligned}
F_{2}^{\prime}(C) & =\sum_{j=1}^{n_{\text {ext }}-1}\left(u_{j}-\mu_{\text {ext } 0}\right)^{2}+\frac{\Delta^{2}}{n_{\text {ext }}-1}+2 \frac{\Delta}{n_{\text {ext }}-1}(-\Delta) \\
& =\sum_{j=1}^{n_{\text {ext }}}\left(u_{j}-\mu_{\text {ext } 0}\right)^{2}-\Delta^{2}-\Delta^{2} /\left(n_{\text {ext }}-1\right) \\
& =\sum_{j=1}^{n_{\text {ext }}}\left(u_{j}-\mu_{\text {ext } 0}\right)^{2}-\Delta^{2} n_{\text {ext }} /\left(n_{\text {ext }}-1\right)
\end{aligned}
$$

The change to the external region is then $\left[-\Delta^{2} n_{\text {ext }} /\left(n_{\text {ext }}-1\right)\right]$.

The coefficient is greater than 1 , but for $\Delta$, we have:

$u_{n}-\mu_{e x t 0}=u_{n}-\frac{u_{n}+\sum_{j=1}^{n_{e x t}-1} u_{j}}{n_{\text {ext }}}=\frac{n_{\text {ext }}-1}{n_{\text {ext }}} u_{n}-\frac{\sum_{j=1}^{n_{e x t}-1} u_{j}}{n_{\text {ext }}}$

So the maximum of $\Delta$ is achieved when $u_{n}=1$ (normalized) and $u_{j}=0$ for others. The external change should satisfy:

$$
C_{e x t}=\frac{n_{e x t}-1}{n_{e x t}}\left(u_{n}-\mu_{\text {ext } 0}\right)^{2} \leq \frac{n_{e x t}}{n_{e x t}-1}\left(\frac{n_{e x t}-1}{n_{e x t}}\right)^{2}=\frac{n_{e x t}-1}{n_{e x t}}<1
$$

Similarly, we can derive the change of the internal factor caused by the movement, which is given by

$C_{\text {int }}=\left[n_{\text {int }} /\left(n_{\text {int }}+1\right)\right] \cdot\left(u_{n}-\mu_{\text {into }}\right)^{2}$

The sum of the two changes gives equation (10). Since the absolute values of both terms fall in the range [0 1) for normalized images, the value range of the regional force is $(-1$ 1 ), which can be directly applied to the formula. 